\title{
Influence of Coulomb Interactions on the Properties of Induced Pairing Model
}

\author{
W.R. Czart and St. Robaszkiewicz \\ Institute of Physics, Adam Mickiewicz University \\ Umultowska 85, 61-614 Poznań, Poland
}

(Received May 18, 2001)

\begin{abstract}
We study the superconducting properties of a model of coexisting itinerant carriers and local pairs with finite binding energy, taking into account the effects of Coulomb (density-density) and direct pair hopping interactions. The evolution of the phase diagrams and superfluid characteristics with electron concentration, interaction parameters and the relative position of the bands is examined. The model is found to exhibit several kinds of superconducting behaviors ranging from the BCS-like to the local-pair-like. The relevance of the obtained results to the interpretation of experimental data for the doped bismuthates $\left(\mathrm{Ba}_{1-x} \mathrm{~K}_{x} \mathrm{BiO}_{3}\right.$ and $\left.\mathrm{BaPb}_{1-x} \mathrm{BiO}_{3}\right)$ is pointed out.
\end{abstract}

PACS numbers: $74.20 .-\mathrm{z}, 74.20 . \mathrm{Mn}, 71.28 .+\mathrm{d}, 74.25 . \mathrm{Ha}$

\section{Introduction}

In Ref. [1] we have reported preliminary results concerning electromagnetic and thermodynamic properties of the model introduced in Ref. [2] and called the induced pairing model, which describes the system of coexisting itinerant carriers (c-subsystem) and local pairs with finite binding energy ( $d$-subsystem) and which is a generalization of the (hard-core) boson-fermion model [3-9].

Here we present further results on this subject and examine the effects of Coulomb (density-density) interactions and the direct pair hopping interaction which were not considered in the previous work. We will determine the phase diagrams and superconducting characteristics of the system considered as a function of total electron concentration $\left(n=n_{c}+n_{d}\right)$, interactions and the relative position of the bands $\left(\Delta_{0}\right)$ within the approach which treats the on site interaction of localized electrons $U_{d}$ exactly, and the remaining interactions within the broken symmetry Hartree-Fock approximation (HFA). 


\section{General formulation}

The effective Hamiltonian of coexisting localized $d$-electrons and itinerant $c$-electrons analyzed in the present work, taking into account the orbital coupling of electrons to the external magnetic field, can be written as

$$
H=H_{d}+H_{c}+H_{c d},
$$

where

$$
\begin{aligned}
& H_{d}=\sum_{i}\left(E_{d}-\mu\right) n_{i}^{d}+U_{d} \sum_{i} n_{i \uparrow}^{d} n_{i \downarrow}^{d}-\frac{1}{2} \sum_{i j}\left(J_{i j} \exp \left(\mathrm{i} 2 \Phi_{i j}\right) \rho_{i}^{+} \rho_{j}^{-}+\text {h.c. }\right) \\
& H_{c}=t \sum_{i j \sigma}\left(\exp \left(\mathrm{i} \Phi_{i j}\right) c_{i \sigma}^{+} c_{j \sigma}+\text { h.c. }\right)-\sum_{i} \mu n_{i}^{c}+\frac{1}{2} \sum_{i j} V_{i j}^{c} n_{i}^{c} n_{j}^{c}, \\
& H_{c d}=\frac{I_{0}}{2 N} \sum_{i k k^{\prime}}\left(\exp \left[\mathrm{i}\left(k-k^{\prime}\right) R_{i}\right] c_{k \uparrow}^{+} c_{\left.-k^{\prime}\right\rfloor}^{+} \rho_{i}^{-}+\text {h.c. }\right)+\frac{1}{2} \sum_{i j} V_{i j}^{c d} n_{i}^{c} n_{j}^{d},
\end{aligned}
$$

$n_{i}^{c}=\sum_{\sigma} c_{i \sigma}^{+} c_{i \sigma}, \quad n_{i}^{d}=\sum_{\sigma} d_{i \sigma}^{+} d_{i \sigma}, \quad \rho_{i}^{+}=\left(\rho_{i}^{-}\right)^{+}=d_{i \uparrow}^{+} d_{i \downarrow}^{+}, E_{d}$ measures the relative position of $d$-level with respect to the bottom of the $c$-electron band $\epsilon_{k}^{c}$ in the absence of interactions, $\mu$ is the chemical potential which ensures that a total number of particles is constant, i.e.

$$
n=n_{c}+n_{d}=\left(\sum_{i}\left\langle n_{i}^{c}\right\rangle+\sum_{i}\left\langle n_{i}^{d}\right\rangle\right) / N
$$

$U_{d}$ is the effective on-site density interaction between $d$-electrons, $J_{i j}$ is the intersite charge exchange interaction of $d$-electrons (pair hopping), $t$ is the hopping integral for $c$-electrons and $I_{0}$ is the intersubsystem charge exchange coupling. $V_{i j}^{c}=U_{c}^{0} \delta_{i j}+V_{i j}^{c}(i \neq j)$ and $V_{i j}^{c d}$ are the Coulomb interactions between $c$-electrons and those between $c$ - and $d$-electrons, respectively. The Peierls factors in (2) and (3) account for the coupling of electrons to the magnetic field via its vector potential $\boldsymbol{A}(\boldsymbol{r}), \Phi_{i j}=(-e / \hbar c) \int_{R_{i}}^{R_{j}} \mathrm{~d} \boldsymbol{r} \boldsymbol{A}(\boldsymbol{r})$, and $e$ is the electron charge.

In analysis we have used, as in our previous works [1, 2], the variational approach which treats the on-site interaction term $U_{d}$ exactly [10] and the intersubsystem and intersite interactions within the broken symmetry HFA [4]. For $\boldsymbol{A}=0$ the resulting expression for the free energy of the superconducting phase $(S)$ is

$$
\frac{F_{0}^{S}}{N}=-\frac{2}{\beta N} \sum_{k} \ln \left(2 \cosh \frac{\beta A_{k}}{2}\right)-\frac{1}{\beta} \ln 2 Z_{d}+\mu\left(n_{d}+n_{c}\right)-2 \mu+C,
$$

where

$$
\begin{aligned}
C= & J_{0}\left(\rho_{0}\right)^{2}+2\left|I_{0}\right| x_{0} \rho_{0}-U_{c}^{0}\left(x_{0}\right)^{2}+\left(U_{c} / 2+U_{c d}\right) n_{c}-\frac{1}{4} U_{c} n_{c}^{2} \\
& -U_{c d} n_{c} n_{d}+U_{c d} n_{d}+\Delta_{0}, \\
A_{k}= & \left(\lambda_{k}^{2}+\left|U_{c}^{0} x_{0}-I_{0} \rho_{0}\right|^{2}\right)^{1 / 2}, \quad \Delta=\sqrt{\bar{\mu}^{2}+\left(J_{0} \rho_{0}+\left|I_{0}\right| x_{0}\right)^{2}}
\end{aligned}
$$




$$
\begin{aligned}
& Z_{d}=\exp \left(\beta U_{d} / 2\right)+\cosh \beta \Delta, \quad \lambda_{k}=\epsilon_{k}^{c}-\tilde{\mu}, \\
& \Delta_{0}=E_{d}+U_{d} / 2, \quad J_{0}=\sum_{j \neq i} J_{i j}, \quad \beta=\left(k_{\mathrm{B}} T\right)^{-1}, \\
& \bar{\mu}=\mu-\Delta_{0}-U_{c d} n_{c}, \quad \tilde{\mu}=\mu-\frac{1}{2} U_{c} n_{c}-U_{c d} n \\
& \rho_{0}=\frac{1}{N} \sum\left\langle\rho_{i}^{+}\right\rangle \quad \text { and } \quad x_{0}=\frac{1}{N} \sum_{k}\left\langle c_{k \uparrow}^{+} c_{-k \downarrow}^{+}\right\rangle
\end{aligned}
$$

are the order parameters for $d$ - and $c$-subsystems, respectively. $\epsilon_{k}^{c}=-t \gamma_{k}, \gamma_{k}=$ $2 \sum_{\alpha} \cos k_{\alpha}, \alpha=x, y, \ldots, U_{c}^{0}=V_{i i}^{c}, U_{c}=2 V_{q=0}^{c}, U_{c d}=V_{q=0}^{c d}$, where $V_{q}^{c(c d)}=$ $\sum_{j} V_{i j}^{e(c d)} \exp \left[\mathrm{i} \boldsymbol{q} \cdot\left(\boldsymbol{R}_{i}-\boldsymbol{R}_{j}\right)\right]$.

Upon minimizing this free energy with respect to the variables $\rho_{0}, 2 x_{0}$, and $\mu$ we obtain the following self-consistent equations:

$$
\begin{aligned}
& x_{0}=\left(U_{c}^{0} x_{0}-\left|I_{0}\right| \rho_{0}\right) \frac{1}{2 N} \sum_{k} \frac{1}{A_{k}} \tanh \frac{\beta A_{k}}{2}, \\
& \rho_{0}=\left(J_{0} \rho_{0}+\left|I_{0}\right| x_{0}\right) \frac{\sinh \beta \Delta}{2 \Delta p Z_{d}}, \\
& n=n_{c}+n_{d},
\end{aligned}
$$

where $n_{c}$ and $n_{d}$ are given by

$$
\begin{aligned}
& n_{c}-1=-\frac{1}{N} \sum_{k} \frac{\epsilon_{k}^{c}-\tilde{\mu}}{A_{k}} \tanh \frac{\beta A_{k}}{2}, \\
& n_{d}-1=\frac{\bar{\mu} \sinh \beta \Delta}{\Delta Z_{d}} .
\end{aligned}
$$

The energy gaps in the c-electron spectrum and the $d$-electron spectrum are given by $E_{g}^{c}(T)=\min \left(2 A_{k}\right)$ and $E_{g}^{d}(T)=\Delta$, respectively, whereas $E_{g}^{\min }(T)=$ $\min \left\{E_{g}^{c}(T), E_{g}^{d}(T)\right\}$ defines the minimum energy gap. The free energy of the normal $(N)$ state $F_{0}^{N}$ is obtained from (6) by putting $x_{0}=\rho_{0}^{x}=0$, and with $\mu$ given by $\partial F_{0}^{N} / \partial \mu=0$.

For a weak static potential the expectation value of the Fourier transform of the total current operator can be obtained from the linear response theory as

$$
J_{\alpha}(\boldsymbol{q}, \boldsymbol{\omega})=N \frac{c}{4 \pi} \sum_{\alpha^{\prime}}\left[\delta_{\alpha \alpha^{\prime}} K^{\mathrm{dia}}+K_{\alpha \alpha^{\prime}}^{\mathrm{para}}(\boldsymbol{q}, \boldsymbol{\omega})\right] A_{\alpha^{\prime}}(\boldsymbol{q}, \boldsymbol{\omega})
$$

and the diamagnetic part of kernel has the following form:

$$
K_{\alpha}^{\text {dia }}=K_{c}+K_{d}
$$

where 


$$
\begin{aligned}
K_{c} & =\frac{8 \pi e^{2}}{\hbar^{2} c^{2} a} \frac{1}{N}|t| \sum_{\boldsymbol{k} \sigma}\left\langle c_{\boldsymbol{k} \sigma}^{+} c_{k \sigma}\right\rangle \cos \left(k_{\alpha}\right) \\
& =\frac{8 \pi e^{2}}{\hbar^{2} c^{2} a N}|t| \sum_{\boldsymbol{k}}\left[1-\frac{\lambda_{\boldsymbol{k}}}{A_{\boldsymbol{k}}} \tanh \left(\beta A_{k} / 2\right)\right] \cos k_{\alpha}, \\
K_{d} & =\frac{32 \pi e^{2}}{\hbar^{2} c^{2} a} \frac{1}{z N}\left\langle-\sum_{k} J_{k} \rho_{k}^{+} \rho_{k}^{-}\right\rangle=\frac{32 \pi e^{2}}{\hbar^{2} c^{2} a}\left(-\frac{J_{0}\left(\rho_{0}\right)^{2}}{z}\right),
\end{aligned}
$$

$z$ is the number of nearest neighbors $(z=2$ for $d=1$ chain, $z=4$ for $d=2$ square lattice, $z=6$ for simple cubic lattice), $a$ is the lattice constant. $\rho_{\boldsymbol{k}}^{ \pm}$, and $J_{\boldsymbol{k}}$ are the space-Fourier transform of $\rho_{i}^{ \pm}$and $J_{i j}$, respectively, and $\rho_{0}, x_{0}$, and $\mu$ are determined by Eqs. (11-13).

In the static limit and $q \rightarrow 0$ for the transverse part of the paramagnetic kernel we obtain

$$
K_{x x}^{\text {para }}(\omega=0)=\frac{8 \pi e^{2} t^{2}}{\hbar^{2} c^{2} a} \frac{1}{N} \sum_{k} \frac{\sin ^{2} k_{x}}{k_{\mathrm{B}} T \cosh ^{2}\left(\beta E_{k} / 2\right)} .
$$

In the London superconductors the magnetic field penetration depth $\lambda$ is determined by the transverse part of the total kernel in the static limit

$$
\lambda=\left\{-K^{\text {dia }}-K_{x x}^{\text {para }}(\omega=0)\right\}^{-1 / 2} .
$$

Using the value of the penetration depth and the difference of the free energy between $N$ and $S$ phase one is able to determine the thermodynamic critical field $H_{\mathrm{C}}$, the Ginzburg-Landau (G-L)correlation length $\xi$ and the Ginzburg ratio $\kappa$ as $H_{\mathrm{C}}^{2}(T)(8 \pi)=\left(F^{N}(T)-F^{S}(T)\right) /\left(N a^{3}\right), \xi=\Phi_{0} /\left(2 \pi \sqrt{2} \lambda H_{\mathrm{C}}\right), \kappa=\lambda / \xi$, where $\Phi_{0}=h c / 2 e$, and to obtain the estimations for the critical fields $H_{c 1} \simeq(\ln \kappa / \kappa) H_{\mathrm{C}}$ and $H_{c 2} \simeq \Phi_{0} / 2 \pi \xi^{2}$.

From Eqs. (11-13) one can calculate the HFA transition temperature $T_{c}$ at which $x_{0} \rightarrow 0$ and $\rho_{0} \rightarrow 0$. The $T_{\mathrm{c}}$ is given by a set of equations

$$
\left\{\begin{array}{c}
\frac{1}{2}\left(J_{0}-I_{0}^{2} \frac{S}{1-U_{c}^{0} S}\right) B=1, \\
n-2=\bar{\mu} B-G
\end{array}\right.
$$

where

$$
\begin{aligned}
B & =\frac{\sinh \beta_{c} \bar{\mu}}{\bar{\mu}\left[\exp \left(\frac{\beta_{c}}{2} U_{d}\right)+\cosh \beta_{c} \bar{\mu}\right]}, \quad G=\frac{1}{N} \sum \tanh \frac{\beta_{c}}{2}\left(\epsilon_{k}^{c}-\widetilde{\mu}\right), \\
S & =\frac{1}{2 N} \sum \frac{\tanh \frac{\beta_{c}}{2}\left(\epsilon_{k}^{c}-\widetilde{\mu}\right)}{\epsilon_{k}^{c}-\widetilde{\mu}} .
\end{aligned}
$$




\section{Results of numerical solution and discussion}

We have performed an extended numerical analysis of Eqs. (6-22) both at $T=0$ and for $T>0$, taking a rectangular density of states for $c$-electrons $\left(N(\epsilon)=1 / 2 D\right.$ for $0<\epsilon_{k}^{c}<2 D$, and 0 otherwise, with $2 D$ denoting an effective $c$-bandwidth). In the following we summarize the main results, concentrating on the phase diagrams and basic superconducting characteristics, derived for several most representative cases.

\subsection{Ground state diagrams}

In the model considered there are two types of mechanisms which can lead to superconductivity. The first one is due to the direct local pair hopping interaction $J$ and involves exclusively the narrow band subsystem. For $U_{d}<0$ it gives rise to a superconductivity analogous to superfluidity of ${ }^{4} \mathrm{He}$.

The second one is due to the intersubsystem charge exchange $I_{0}$. This leads to a superconducting state involving both subsystems, in which the single-particle spectrum of the wide band electrons opens up a gap around the position of the narrow band of electrons in pair states [2-8].

Figures 1 and 2 show the evolution of the ground state phase diagrams as a function of $n$ and $\Delta_{0} / D$ obtained for $-\infty \leq U_{d} \leq 0$ and several fixed values of $J_{0}, I_{0}, U_{c}$, and $U_{c d}$.

Depending on the relative concentration of " $c$ " and " $d$ " electrons, which is determined by $\Delta_{0}=E_{0}+U_{d} / 2$, the interactions and $n$, we distinguish three essentially different physical situations. In the most interesting case of $I_{0} \neq 0$ (Fig. 2) it will be: (i) $d$-regime, $n_{d} \gg n_{c}$ (LP), (ii) $d+c$ regime, $2>n_{c}, n_{d}>0$ (MIXED), (iii) $c$-regime, $n_{c} \gg n_{d}$ (BCS). In $d$-regime the superconducting properties of the system are analogous (at least for $U_{d} \leq 0$ ) to those of pure local-pair superconductor, whereas in c-regime the situation is similar to the BCS case: pairs of " $c$ " electrons with opposite momenta and spins are exchanged via virtual transitions into empty $d$-levels. In $d+c$ regime the system shows features which are intermediate between those of pure local-pair superconductor and those of classical BCS systems, even in the strong attraction $\left(U_{d} \ll 0\right)$ case. This concerns, among others, the gap in the single-particle excitation spectrum for $c$-electrons, the ratio $k_{\mathrm{B}} T_{c} / E_{g}(0)$, the electromagnetic coherence length as well as the thermodynamic critical field (see Sec. 3.2).

As for the evolution of the superconducting properties with an increasing total number of carriers, there are three possible types of change-over: (i) BCS $\longrightarrow$ "Crossover" (MIXED) $\longrightarrow \mathrm{BCS}$, (ii) BCS $\longrightarrow \mathrm{LP}$, and (iii) LP $\longrightarrow$ BCS (comp. Fig. 2). Only in the case when the local pair level is deeply located below the bottom of the wide $c$ band $\left(\Delta_{0} \ll 0\right)$, the system remains in $d$-regime for any $n \leq 2$.

Let us also observe that with the increase in $\Delta_{0} / D$ at fixed $n$ there are two sequences of change-overs possible: LP $\rightarrow$ MIXED $\rightarrow$ BCS (if $n<2$ ) and BCS $\rightarrow$ $\mathrm{MIXED} \rightarrow \mathrm{LP}$ (if $n>2$ ). 

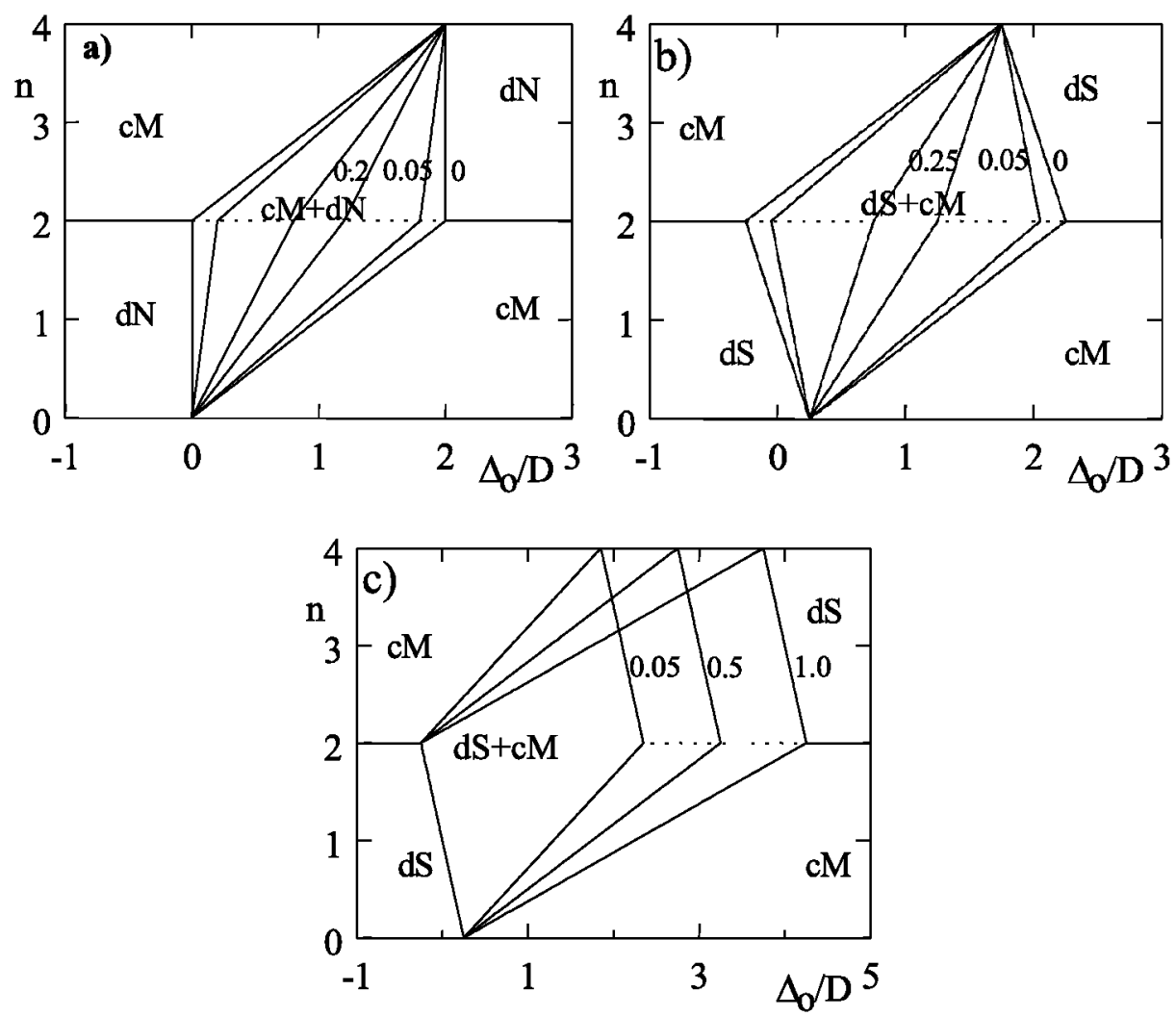

Fig. 1. Ground state diagrams of the model (1) as a function of $n$ and $\Delta_{0} / D$ plotted for $-\infty \leq U_{d} \leq 0, I_{0}=0$ and different values of $J_{0} / 2 D, U_{c} / 2 D, U_{c d} / 2 D$, showing the effects of increasing Coulomb interactions $U_{c}$ and $U_{c d}$; (a) $J_{0} / 2 D=0, U_{c} / 2 D=0, U_{c d} / 2 D=0$; $0.05 ; 0.2$ (numbers to the curves-n.t.c.); (b) $J_{0} / 2 D=0.25, U_{c} / 2 D=0, U_{c d} / 2 D=0 ; 0.05$; 0.25 (n.t.c.); (c) $J_{0} / 2 D=0.25, U_{c} / 2 D=0.05 ; 0.5 ; 1$ (n.t.c.), $U_{c d} / 2 D=0$. Notation: $c M$ (metallic state of $c$-electrons), $d N$ (normal state of $d$-electrons), $d S$ (superconducting state of $d$-electrons).

In contrast to the change-overs MIXED $\longrightarrow$ BCS, which are quite sharp, the evolution between the LP and MIXED regimes for $I_{0} \neq 0$ is rather smooth (comp. Figs. 4,5 ) and therefore the LP/MIXED boundary cannot be precisely located on the $n$ vs. $\Delta_{0} / D$ diagrams.

In the case $I_{0}=0, J_{0} \neq 0$, for which the ground state diagrams are shown in Fig. 1, the situation is simpler. The superconducting state is restricted to the regions marked by $d S$ and $d S+c M$, and all the phase boundaries on the diagram are well defined. At the border $d S+c M / d S: n_{c} \rightarrow 0$ (for $n<2$ ), or $n_{c} \rightarrow 2$ (for $n>2$ ), whereas at the border $d S+c M / c M: n_{d} \rightarrow 0$ (for $n<2$ ), or $n_{d} \rightarrow 2$ (for $n>2)$. 

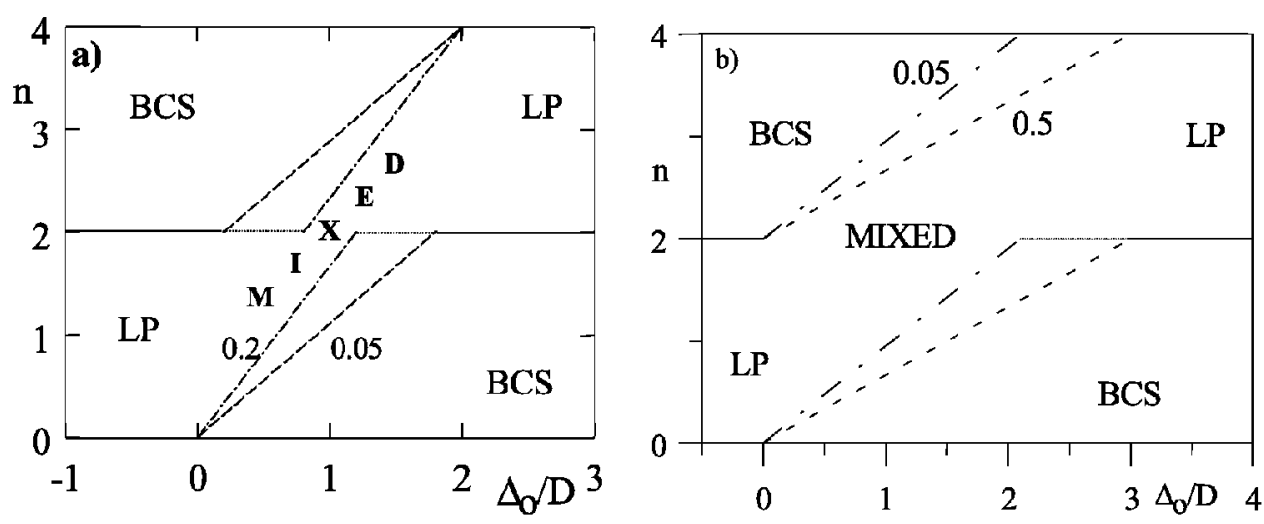

Fig. 2. Ground state diagrams as a function of $n$ and $\Delta_{0} / D$ plotted for $-\infty \leq U_{d} \leq 0$, $J_{0}=0, I_{0} / 2 D=0.1$ and different values of $U_{c d} / 2 D, U_{c} / 2 D ;$ (a) $U_{c d} / 2 D=0.05$, 0.2 (numbers to the curves-n.t.c.), $U_{c} / 2 D=0$, (b) $U_{c} / 2 D=0.05,0.5$ (n.t.c.), $U_{c d} / 2 D=0$. BCS, LP, and MIXED denote superconducting state of predominantly $c$-electrons, $d$-electrons and coexisting $c+d$ electrons, respectively. By dashed lines we mark the values of $\Delta_{0} / D$ and $n$ for which $n_{d}$ or $2-n_{d}$ become vanishingly small $\left(n_{d} / n=10^{-5}\right)$ defining in this way the approximate border MIXED/BCS.

As it follows from the diagrams shown in Figs. 1c, $2 b$ the intrasubsystem Coulomb interaction $U_{c}$ enlarges the coexistence region of local pairs and wide band electrons $\left(n_{d} \neq 0, n_{c} \neq 0\right.$ ) expanding it towards higher values of $\Delta_{0} / D$, both, in the case of $I_{0}=0, J_{0} \neq 0$ (cf. Fig. 1c) as well as in the case of $J_{0}=0$, $I_{0} \neq 0$ (cf. Fig. 2b), and the concentrations of both types of particles change smoothly with varying $\Delta_{0}$.

The influence of the intersubsystem Coulomb repulsion $U_{c d}$ is more drastic as it tends to reduce the coexistence region (cf. Figs. 1a, b, for $I_{0}=0$, and Fig. 2a, for $I_{0} \neq 0$ ). For $U_{c d} / 2 D<0.5, D$ being the half-bandwidth of $c$-electron band, changes in the concentrations of both types of carriers, $n_{c}$ and $n_{d}$ versus $\Delta_{0}$ are smooth. On the other hand for $U_{c d} / 2 D>0.5$ discontinuous changes in $n_{c}$ and $n_{d}$ with variation of $\Delta_{0}$ and (or) temperature can take place, and the $d+c$ (MIXED) regime can be completely suppressed. It remains an open question whether such behavior in the case of strong intersubsystem Coulomb repulsion is an inherent property of the model or if it is due to the approximation used.

\subsection{Superconducting properties}

Let us first conclude the evolution of basic superfluid characteristics with the particle concentration $n$ and $\Delta_{0} / D$ at $T=0, U_{d} \leq 0$. Examples of the plots of $H_{c}^{2}, \xi, 1 / \lambda^{2}$ and $\kappa$ vs. $n$ and vs. $\Delta_{0} / D$ are shown in Figs. 3 and 4 , respectively, for the fixed values of $I_{0}$ and $J_{0}\left(U_{c}=U_{c d}=0\right)$. 

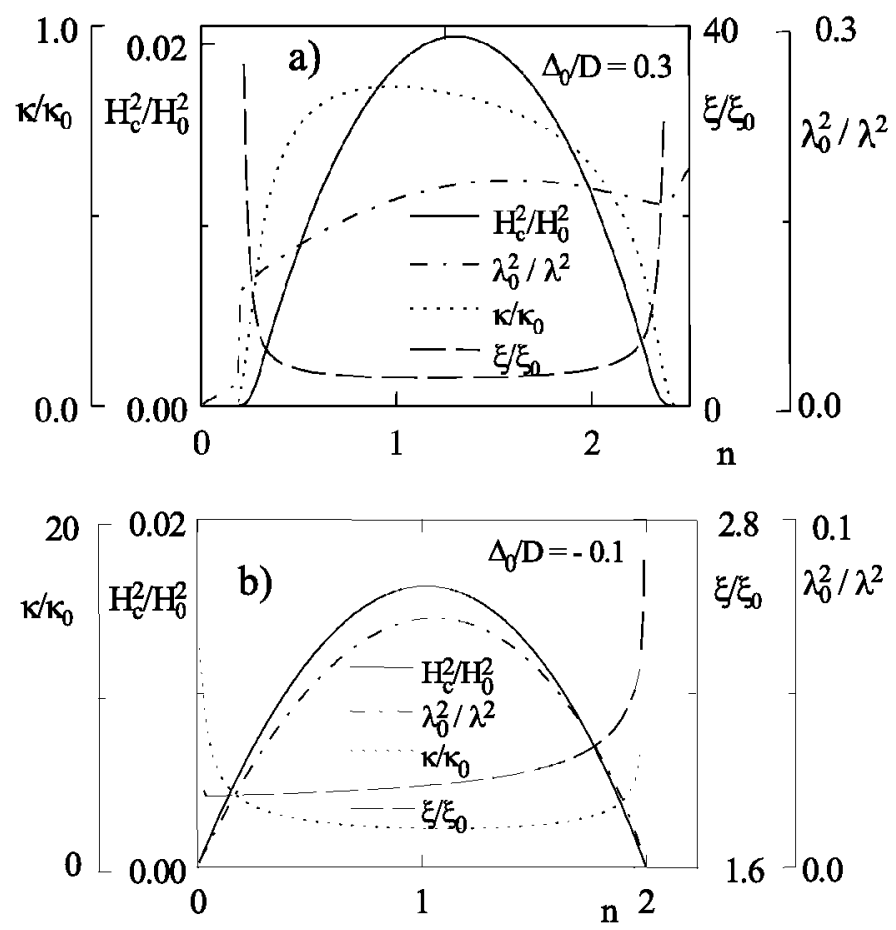

Fig. 3. Concentration dependences of $H_{c}^{2}, \xi, 1 / \lambda^{2}$, and $\kappa$ at $T=0$ for $I_{0} / 2 D=0.1, J_{0} / 2 D=0.05,-\infty \leq U_{d} \leq 0, U_{c}=U_{c d}=0$, (a) $\Delta_{0} / D=+0.3$, (b) $\Delta_{0} / D=-0.1 . H_{0}^{2}=16 \pi D / a^{3}, \quad \xi_{0}=a / 4 \sqrt{2}, \lambda_{0}^{2}=\hbar^{2} c^{2} a z / 16 \pi e^{2} D$, $\kappa_{0}=(\sqrt{2} \hbar c / \sqrt{\pi} e)(1 / \sqrt{a D}), D=z t$.

In Fig. 3 we show the evolution of the thermodynamical critical field $H_{c}$, the coherence length $\xi$ and the Ginzburg ratio $\kappa=\lambda / \xi$ and $1 / \lambda^{2}$ with electron concentration $n=n_{d}+n_{c}$, for two fixed values of $\Delta_{0} / D$ and $0 \leq U_{d}$.

For $\Delta_{0} / D=0.3$ and $I_{0} \neq 0$ (Fig. 3a) upon increasing $n$ the system exhibits two change-overs: from the $c$-regime (BCS) $\left(n_{c} \gg n_{d} \approx 0\right)$ into the $c+d$ (MIXED) regime and then again into the $c$-regime (BCS) $\left(n_{c} \approx n-2, n_{d} \approx 2\right)$. Notice only a weak $n$-dependence of $\lambda, \xi$, and $\kappa$ in a wide range of $n$. This is due to a strong pinning of $\mu$ around the $d$-level $\left(\Delta_{0}\right)$ in the $c+d$ regime, which yields $n_{c}$ to be almost $n$-independent.

For $\Delta_{0} / D=-0.1$ (Fig. 3b) $n_{d} \gg n_{c}$ (except $n \approx 2$ and $n>2$ ) and electromagnetic characteristics for $n<2$ are dominated by the $d$-subsystem. With increasing $n$ the system exhibits a single change-over from the predominantly $d$-regime (LP) directly into the $c$-regime $(\mathrm{BCS})$ at $n \approx 2$. One can observe it in the behavior of $\xi$, which substantially increases near $n=2$. In comparison to the former case $\xi$ is strongly reduced, whereas $\lambda$ and $\kappa$ - strongly enhanced ( $\kappa$ up to 


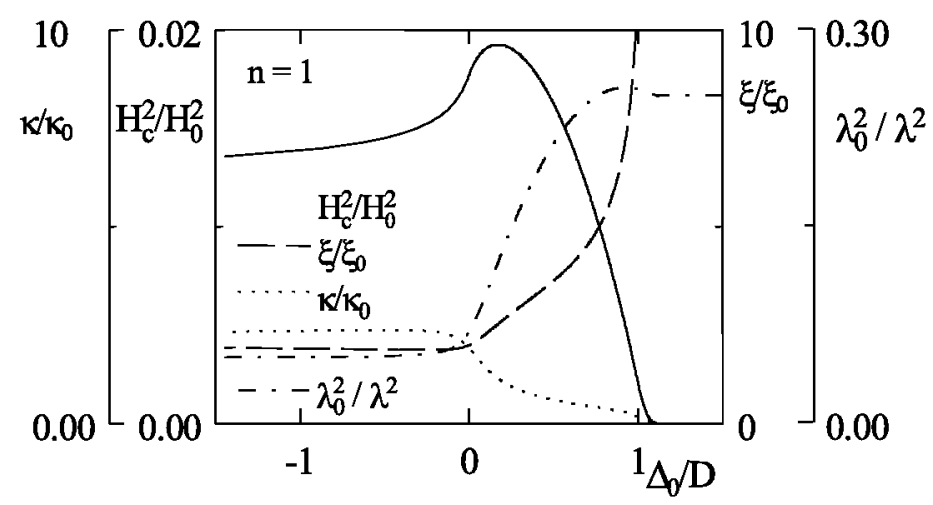

Fig. 4. The plots of $H_{c}^{2}, \xi, 1 / \lambda^{2}$ and $\kappa$ as a function of $\Delta_{0} / D$ at $T=0$ for $n=1$, $I_{0} / 2 D=0.1, J_{0} / 2 D=0.05,-\infty \leq U_{d} \leq 0, U_{c}=U_{c d}=0 . H_{0}^{2}=16 \pi D / a^{3}, \xi_{0}=a / 4 \sqrt{2}$, $\lambda_{0}^{2}=\hbar^{2} c^{2} a z / 16 \pi e^{2} D, \kappa_{0}=(\sqrt{2} \hbar c / \sqrt{\pi} e)(1 / \sqrt{a D}), D=z t$.

the factor 40) for $n<2$. Also their concentration dependences are very different (comp. Fig. 3b with Fig. 3a).

The crossover between the local-pair-like and BCS-like behavior is clearly seen in the variation of $\xi, \kappa$, and $1 / \lambda^{2}$ with increasing $\Delta_{0}$ (cf. Fig. 4). For $\Delta_{0} \leq 0$, $\xi(\kappa)$ is very small (large), indicating extreme type II superconductivity, and $\xi(\kappa)$ rapidly increases (decreases) as one approaches the $c$-regime at $\Delta_{0} / D \approx n$. The London penetration depth $\lambda(0)$ being very large in the local pair limit $\left(\Delta_{0}<0\right)$ decreases with increasing $\Delta_{0}$ in $d+c$ regime, whereas in $c$-regime it remains almost constant.

Figure 5 shows the variation of the ratio $2 k_{\mathrm{B}} T_{\mathrm{c}} / E_{g}^{\min }(0)$ as a function of $\Delta_{0} / D$ for $n=1, J_{0}=0$ and a fixed value of $I_{0} / 2 D$ in the cases of $U_{d} \rightarrow-\infty$ and $U_{d}=0$. As we see the ratio approaches the BCS value in $c$-regime and it strongly

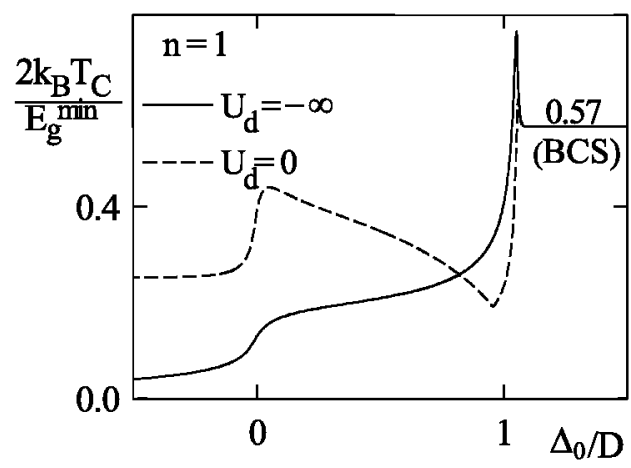

Fig. 5. The variation of the ratio $2 k_{\mathrm{B}} T_{c} / E_{g}^{\min }(0)$ as a function of $\Delta_{0} / D$ for $U_{d}=-\infty$ and $U_{d}=0$ at $n=1$, where $E_{g}^{\min }(0)=\min \left\{E_{g}^{c}(0), E_{g}^{d}(0)\right\}$, plotted for $I_{0} / 2 D=0.1$, $J_{0}=U_{c}=U_{c d}=0$. 
decreases as the concentration of $d$-electrons increases above that of wide band electrons with decreasing $\Delta_{0}$.

The Coulomb interactions $U_{c d}, U_{c}$ can strongly influence the concentration dependences of superconducting characteristics both in the local pair $(d)$ regime as well as in the mixed $(d+c)$ regime.

In Fig. 6 we show the evolution of the London penetration depth $\left(1 / \lambda^{2}\right)$ at $T=0$ as a function of total electron concentration $n$ for various values of intersubsystem Coulomb repulsion $U_{c d}$ and two fixed values of $\Delta_{0} / D$, whereas Fig. 7 shows examples of the plots of $1 / \lambda^{2}$ as a function of $\Delta_{0}$ for the fixed values of $n$ and $U_{c d}$. Both these figures are calculated for $I_{0} / 2 D=0.1, J_{0}=0$,
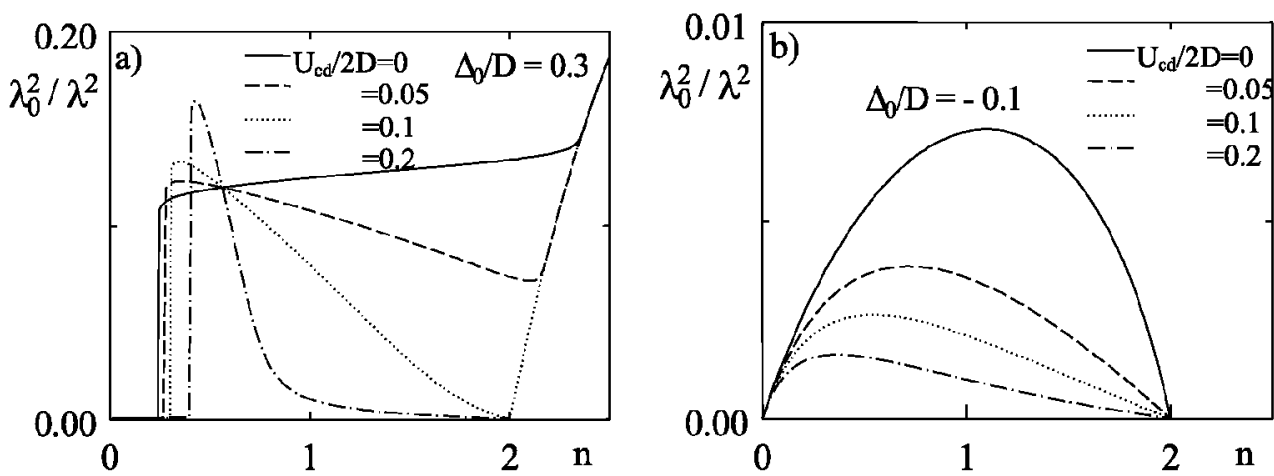

Fig. 6. The inverse square of penetration depth $\lambda^{-2}$ at $T=0$ as a function of total concentration of carriers $n$ for different values of $\Delta_{0} / D$ and interaction $U_{c d} / 2 D$. $I_{0} / 2 D=0.1, J_{0}=U_{c}=0,-\infty \leq U_{d} \leq 0, U_{c d} / 2 D=0,0.05,0.1,0.2$. $\lambda_{0}^{2}=\hbar^{2} c^{2} a z / 16 \pi e^{2} D ;$ (a) $\Delta_{0} / D=0.3$, (b) $\Delta_{0} / D=-0.1$.
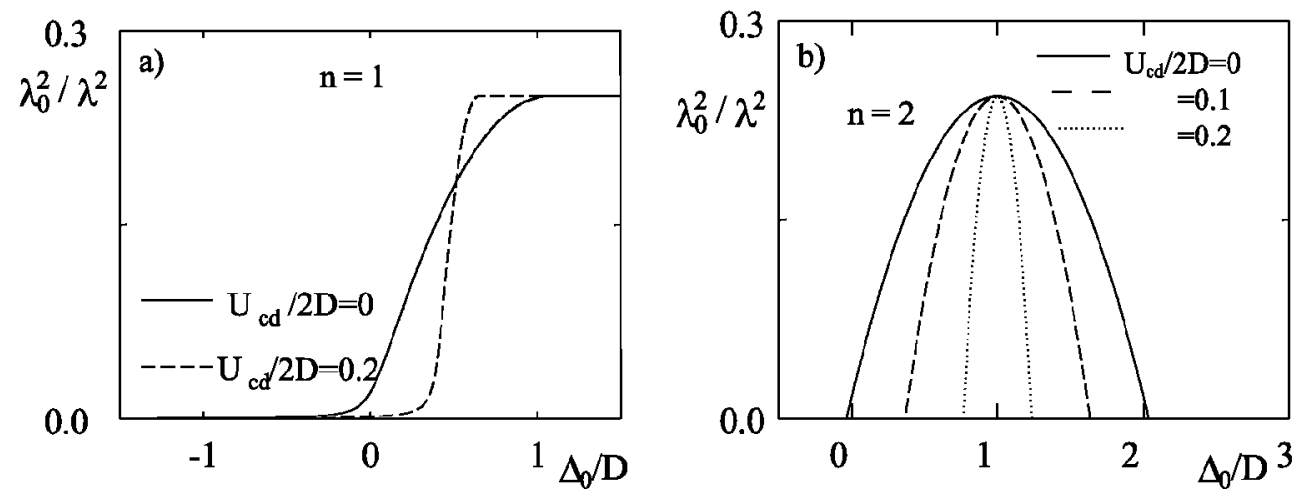

Fig. 7. The inverse square of penetration depth $\lambda^{-2}$ at $T=0$ as a function of $\Delta_{0} / D$ for different values of $n$ and interaction $U_{c d} / 2 D . I_{0} / 2 D=0.1, J_{0}=U_{c}=0,-\infty \leq U_{d} \leq 0$; $\lambda_{0}^{2}=\hbar^{2} c^{2} a z / 16 \pi e^{2} D$; (a) $n=1, U_{c d} / 2 D=0,0.2$, (b) $n=2, U_{c d} / 2 D=0,0.1,0.2$. 
$-\infty \leq U_{d} \leq 0$, as the ground state diagrams given in Fig. 2a. Figure 6a is plotted for $\Delta_{0} / D=0.3$. In this case upon increasing $n$ one observes two change-overs: $\mathrm{BCS} \rightarrow$ MIXED $\rightarrow$ BCS (comp. the diagrams $2 \mathrm{a}$ and Fig. 3a). On the contrary, for $\Delta_{0} / D=-0.1$ (Fig. $6 \mathrm{~b}$ ) the superfluid characteristics for any $n<2$ are dominated by the $d$-subsystem $\left(n_{d} \gg n_{c}\right)$, comp. Figs. $2 \mathrm{a}$ and $3 \mathrm{~b}$. In comparison to the former case $1 / \lambda^{2}$ is strongly reduced (up to the factor 20) and shows a quite different concentration dependence. Also the effects of $U_{c d}$ on $\lambda(n)$ are different in both cases.

The superconducting critical temperature $T_{\mathrm{c}}$ as a function of $\Delta_{0}$ is influenced by $U_{c}$ and $U_{c d}$ in the following way. The repulsive $U_{c}$ shifts the maximum of $T_{\mathrm{c}}$ towards higher values of $\Delta_{0}$. This is simply a consequence of extending the coexistence region of $d$ and $c$ electrons by $U_{c}$ (cf. Figs. 1c, 2b). The effect of $U_{c d}$ is different: there is no essential shift of the maximum $T_{\mathrm{c}}$ versus $\Delta_{0}$, and the coexistence region is reduced. Examples of the plots of $T_{\mathrm{c}}$ vs. $\Delta_{0} / D$ are shown in Fig. 8 for $n=2$ and a few fixed values of $U_{c}$ and $U_{c d}$.

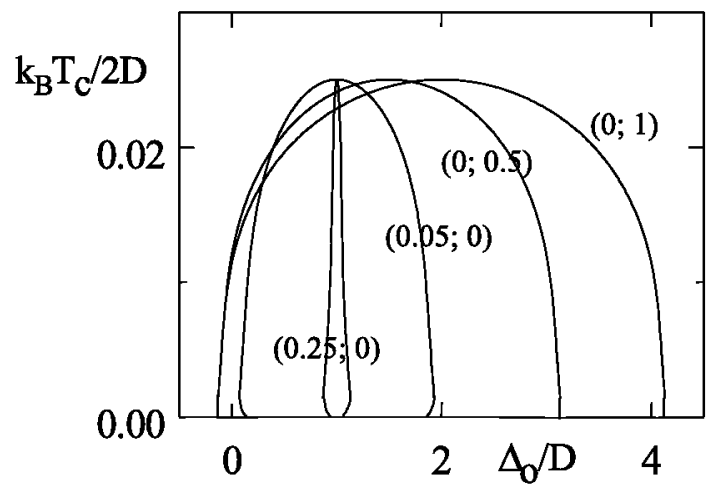

Fig. 8. The superconducting critical temperature as a function of $\Delta_{0} / D$ for $J_{0} / 2 D=$ $0.05, I_{0}=0, n=2, U_{d} / D=-\infty$. The values of $U_{c}$ and $U_{c d}$ are given in the brackets $\left(U_{c d} / 2 D ; U_{c} / 2 D\right)$ next to the curves.

Closing this section let us shortly conclude the effects of on-site density interaction $U_{d}$, being the main factor determining the $d$-pair binding energy $E_{b}^{d}$ (for a more detailed discussion see Refs. [1, 2, 11]).

For $U_{d} \leq 0$, decreasing $\left|U_{d}\right|$ yields a moderate reduction of $T_{c}$ in comparison to the $U_{d}=-\infty$ case (up to a factor 2 for $U_{d} \rightarrow 0$ ). As we see from Fig. 5 the ratio $k_{\mathrm{B}} T_{c} / E_{g}^{\min }(0)$ is substantially enhanced by a decrease in $\left|U_{d}\right|$ in the $d(\mathrm{LP})$ regime and it can be either enhanced or reduced in the $c+d$ (MIXED) regime. In the $c$ regime $k_{\mathrm{B}} T_{c} / E_{g}^{\mathrm{min}}(0)$ remains almost unaffected by $U_{d}$ and it approaches the BCS value (0.57) for any $U_{d} \leq 0$.

The repulsive $U_{d}$ competes both with $I_{0}$ and $J_{i j}$ and its effects on the properties of the system can be much more spectacular than those of $U_{d}<0$. In general, for a weak repulsion the superconducting transition remains continuous, as for 
$U_{d}<0$, however the $d$-pair density exhibits a sharp break at $T_{c}$. The $T_{c}, H_{c}^{2}(0)$, $\kappa$, and (for $I_{0} \neq 0$ ) the ratio $k_{\mathrm{B}} T_{c} / E_{g}^{c}(0)$ is strongly reduced, whereas the $\mathrm{G}-\mathrm{L}$ coherence length is enhanced. The increasing $U_{d}$ changes the nature of the phase transition from a continuous to a discontinuous type, resulting in the tricritical point (TCP), then it suppresses superconductivity for high $n_{d}\left(\left|n_{d}-1\right| \approx 1\right)$. Finally, for a large $U_{d}$ the system remains in the normal state at any temperature and any $n_{d}[2,11]$.

\section{Final remarks}

We have studied the thermodynamic and electromagnetic properties and phase diagrams of an induced pairing model. The model considered assumes arbitrary value of the on-site density interaction between localized $d$-electrons $U_{d}$, which allows investigation of the effects of reduced $d$-pair binding energy. Moreover, it takes into account the Coulomb interactions between itinerant electrons $U_{c}$ and those between $c$ and $d$ electrons $U_{c d}$ as well as the direct pair hopping interaction $J_{i j}$.

Depending on parameters the model is found to exhibit several kinds of superconducting behavior ranging from the BCS-like to the local-pair-like. In particular, for $I_{0} \neq 0, U_{d} \leq 0$ and fixed $n$ the electromagnetic properties like $\xi, H_{c}, \lambda$, $\kappa$ as well as the ratio $k_{\mathrm{B}} T_{\mathrm{c}} / E_{g}^{\min }(0)$ can evolve with the position of LP level $\left(\Delta_{0}\right)$ from LP to "Crossover" (MIXED) and finally to BCS-like regime. There are also three possible types of change-overs with an increasing total number of carriers: $\mathrm{BCS} \longrightarrow \mathrm{MIXED} \longrightarrow \mathrm{BCS}, \mathrm{BCS} \longrightarrow \mathrm{LP}$ and $\mathrm{LP} \longrightarrow \mathrm{BCS}$.

As we found, the Coulomb interactions $U_{c}$ and $U_{c d}$ can substantially change the ground state diagrams of the model and strongly modify the evolution of basic superconducting characteristics with particle concentration and $\Delta_{0}$. The intrasubsystem Coulomb interaction $U_{c}$ enlarges the coexistence region of $c$ and $d$ particles (MIXED regime). On the contrary, the intersubsystem Coulomb repulsion $U_{c d}$ tends to reduce the MIXED region and the range of $n$ in which both types of particles coexist.

There are various groups of materials for which the coexistence of local pair states with itinerant electron states have been either established or suggested and which can be suitable candidates for the present model [3-9, 12]. Among these materials there are also several nonstandard ("exotic") superconductors, including the high- $T_{\mathrm{c}}$ cuprates, the tungsten bronzes and the doped bismuthates $\left(\mathrm{Ba}_{1-x} \mathrm{~K}_{x} \mathrm{BiO}_{3}\right.$ and $\left.\mathrm{BaPb}_{1-x} \mathrm{Bi}_{x} \mathrm{O}_{3}\right)$.

For the bismuthates the experimental results show the existence of $p$-holes together with disproportionated (at least locally) $\mathrm{Bi}^{3+}-\mathrm{Bi}^{5+}$ background $[13,14]$, and the coexistence of itinerant carriers with small bipolarons has been indicated by the Raman spectroscopy data [15]. The existence of local electron pairing is also supported by several photoemission studies, which provide the evidences of a pseudogap behavior in the normal state of these materials [16]. 
Recently Puchkov et al. [17] have analyzed the doping dependence of the optical properties of $\mathrm{Ba}_{1-x} \mathrm{~K}_{x} \mathrm{BiO}_{3}$ in the full doping range $(0<x \leq 0.46)$. In contrast to the cuprates the London penetration depth has been found to be almost doping independent in an extended range of $x$, i.e. it exhibits the behavior analogous to that corresponding to $\Delta_{0} / D=0.3$ in Figs. 3a and $6 \mathrm{a}$. This suggests that the system considered is in the $c+d$ (MIXED) regime for a wide range of doping. Support for such a conclusion is provided by the experimental finding that in doped $\mathrm{BaBiO}_{3}$ the density of the itinerant (Drude) charge carriers does not significantly change with doping [17]. Within our theory such a result is easy to explain by a strong pinning of chemical potential $\mu$ around the $d$-level $\left(\Delta_{0}\right)$ in the MIXED regime, which yields the itinerant carriers concentration $n_{c}$ to be almost $n$-independent.

A more detailed discussion on the relevance of the induced pairing model to the interpretation of experimental data for the cuprates will be given elsewhere [11].

\section{Acknowledgments}

We would like to thank R. Micnas, J. Ranninger, M.C. Ren and P. van Dongen for valuable discussions and comments. This work was supported in part by the State Committee for Scientific Research with the project 2 P03 B037 17.

\section{References}

[1] W. Czart, M. Szkudlarek, S. Robaszkiewicz, Acta Phys. Pol. A 91, 415 (1997).

[2] G. Pawłowski, S. Robaszkiewicz, Mol. Phys. Rep. 12, 191 (1995); idem, Acta Phys. Pol. A 94, 683 (1998).

[3] J. Ranninger, S. Robaszkiewicz, Physica B 135, 468 (1985).

[4] S. Robaszkiewicz, R. Micnas, J. Ranninger, Phys. Rev. B 46, 180 (1987).

[5] R. Micnas, J. Ranninger, S. Robaszkiewicz, Rev. Mod. Phys 62, 113 (1990).

[6] J. Ranninger, J.M. Robin, Physica C 253, 279 (1995); J. Ranninger, J.M. Robin, M. Eschrig, Phys. Rev. Lett. 74, 4027 (1995); P. Devilard, J. Ranninger, Phys. Rev. Lett. 84, 5200 (2000); H.C. Ren, Physica C 303, 115 (1998).

[7] R. Friedberg, T.D. Lee, M.C. Ren, Phys. Rev. B 42, 4122 (1990); ibid. B 45, 10732 (1992); E. Piegari, V. Cataudella, G. Iadonisi, Physica C 303, 273 (1998).

[8] V.B. Geshkenbein, L.B. Ioffe, A.I. Larkin, Phys. Rev. B 55, 3173 (1997); C.P. Enz, Phys. Rev. B 54, 3589 (1996).

[9] R. Micnas, S. Robaszkiewicz, in: High-Tc Superconductivity 1996; Ten Years after the Discovery, Eds. E. Kaldis, E. Liarokapis, K.A. Müller, NATO ASI Series E, Vol. 342, Kluwer Academic Publ., Dordrecht 1997, p. 31 and references therein; R. Micnas, S. Robaszkiewicz, B. Tobijaszewska, J. Supercond. 12, 79 (1999).

[10] S. Robaszkiewicz, G. Pawłowski Physica C 210, 61 (1993); S. Robaszkiewicz, Acta Phys. Pol. A 85, 117 (1994).

[11] W.R. Czart, S. Robaszkiewicz, unpublished. 
[12] J.A. Wilson, Physica C 233, 332 (1994); J.A. Wilson, A. Zahir, Rep. Prog. Phys. 60, 941 (1998).

[13] C.L. Lin, S.L. Qiu, Jie Chen, Myron Strongin, Gang Cao, ChanSoo Jee, J.E. Crow, Phys. Rev. B 39, 9607 (1989).

[14] C.H. Rüscher, H. Heinrich, W. Urland, Physica C 219, 471 (1994); E.S. Hellman, E.H. Hartford Jr., Phys. Rev. B 52, 6822 (1995).

[15] S. Sugai, Solid State Commun. 72, 1187 (1990).

[16] S. Tajima, S. Uchida, A. Masaki, H. Takagi, K. Kitazawa, S. Tanaka, S. Sugai, Phys. Rev. B 35, 696 (1987); J.B. Boyce, F.G. Bridges, T. Claeson, T.H. Geballe, G.G. Li, A.W. Sleight, Phys. Rev. B 44, 6961 (1991); M.A. Karlow, S.L. Cooper, A.L. Kotz, M.V. Klein, P.D. Han, D.A. Payne, Phys. Rev. B 48, 6499 (1993); H. Namatame, A. Fujimori, H. Torii, T. Uchida, Y. Nagata, J. Akimitsu, Phys. Rev. B 50, 13674 (1994).

[17] A. Puchkov, T. Timusk, M.A. Karlow, S.L. Cooper, P.D. Han, D.A. Payne, Phys. Rev. B 54, 6686 (1996). 